\title{
Chiral effective theory of diquarks and the $U_{A}(1)$ anomaly
}

\author{
Masayasu Harada, ${ }^{1}$ Yan-Rui Liu $\odot,{ }^{2}$ Makoto Oka๑, ${ }^{3,4,{ }^{*}}$ and Kei Suzuki ${ }^{3}$ \\ ${ }^{1}$ Department of Physics, Nagoya University, Nagoya, 464-8602, Japan \\ ${ }^{2}$ School of Physics, Shandong University, Jinan 250100, China \\ ${ }^{3}$ Advanced Science Research Center, Japan Atomic Energy Agency, Tokai, Ibaraki 319-1195, Japan \\ ${ }^{4}$ Nishina Center for Accelerator-Based Science, RIKEN, Wako 351-0198, Japan
}

(Received 22 December 2019; accepted 9 March 2020; published 27 March 2020)

\begin{abstract}
The diquark is a strongly correlated quark pair that plays an important role in hadrons and hadronic matter. In order to treat the diquark as a building block of hadrons, we formulate an effective theory of diquark fields with $S U(3)_{R} \times S U(3)_{L}$ chiral symmetry. We concentrate on the scalar $\left(0^{+}\right)$and pseudoscalar $\left(0^{-}\right)$diquarks and construct a linear-sigma-model Lagrangian. It is found that the effective Lagrangian contains a new type of chirally symmetric meson-diquark-diquark coupling that breaks axial $U_{A}(1)$ symmetry. We discuss consequences of the $U_{A}(1)$ anomaly term to the diquark masses as well as to the singly heavy baryon spectrum, which is directly related to the diquark spectrum. We find an inverse mass ordering between strange and nonstrange diquarks. The parameters of the effective theory can be determined by the help of lattice QCD calculations of diquarks and also from the mass spectrum of the singly heavy baryons. We determine the strength of the $U_{A}(1)$ anomaly term, which is found to give a significant portion of the diquark masses.
\end{abstract}

DOI: $10.1103 /$ PhysRevD.101.054038

\section{INTRODUCTION}

Recent developments of hadron spectroscopy has brought a completely new picture of hadrons. In particular, many unconventional hadron resonances have been found and become candidates of multiquark exotic states, which contain more than three quarks [1-4]. $\mathrm{X}(3872)$ is a representative of such states. While it is found in the charmonium $(c \bar{c})$ spectrum, it may not simply be a $c \bar{c}$, but is dominantly a tetraquark state, or a $D \bar{D}^{*}$ molecular resonance. The other unconventional new resonances include charged hidden-charm mesons and hidden-charm pentaquark baryons. It is urgent and important to reveal the composition and dynamics of these states.

In understanding their structures, we need a new dynamics that gives a strong correlation among multiple quarks. Various types of subsystems, or clusters, have been proposed to form exotic multiquark states. A candidate is color-singlet hadrons, which form a bound or resonance states. Color-non-singlet clusters are more exotic and interesting. The simplest one, except for a constituent quark, is a diquark [5-7]. Various models with diquarks

\footnotetext{
Corresponding author. oka@post.j-parc.jp

Published by the American Physical Society under the terms of the Creative Commons Attribution 4.0 International license. Further distribution of this work must maintain attribution to the author(s) and the published article's title, journal citation, and DOI. Funded by SCOAP ${ }^{3}$.
}

as ingredients have been proposed for explaining masses and structures of multiquark hadrons [8-10]. Among many possible quantum numbers, it is known that the scalar $\left(0^{+}\right)$ diquark with color $\overline{3}$ and flavor $\overline{3}$ is strongly favored by quantum chromodynamics (QCD) [11-15], while the axialvector $\left(1^{+}\right)$diquark with color $\overline{3}$ and flavor 6 also appear frequently in hadrons. Color 6 diquarks are interesting also, but they may appear only in the multiquark states.

We consider a diquark effective theory for light quarks $q=(u, d, s)$. It is important to formulate their dynamics based on the chiral symmetry and its spontaneous breaking in QCD. Chiral symmetry plays key roles in understanding the low-lying spectrum of mesons and baryons. In particular, the pion (and the other ground-state pseudoscalar mesons) and its properties have revealed how chiral symmetry is broken in the QCD vacuum. The symmetry constrains the low-energy effective theory of the pion very strongly. In contrast, diquarks show new features and dynamics under the chiral symmetry [10,16-19]. Effective theories of diquarks were also explored in the context of color superconductivity at high density QCD [20,21], where it is shown that the axial $U_{A}(1)$ anomaly plays an important role $[22,23]$.

Chiral symmetry of diquarks is closely related to the chiral representations of heavy-quark $(Q)$ baryons $(Q q q)$, such as singly charmed (cqq) or bottomed (bqq) baryons. In fact, the roles of diquarks are most prominently seen in the singly heavy baryon system [24-27]. Chiral aspects of heavy baryon masses and decays were studied in [28,29]. 
In this paper, we propose a chiral effective theory of scalar and pseudoscalar diquarks, based on linear representations of $S U(3)_{R} \times S U(3)_{L}$ symmetry. Such an effective theory may be applied, once the parameters are determined by the known experimental data, to not only the heavy baryon systems, but also to tetraquarks, and the other multiquark hadrons. We write down a general form of the effective Lagrangian for the scalar diquarks in the form of the linear sigma model. Then the masses of the diquarks are identified at the tree level from the quadratic terms of the Lagrangian.

It is shown that the leading meson-diquark-diquark coupling term breaks axial $U_{A}(1)$ symmetry, while it keeps the chiral symmetry invariant. Such a term is supposed to come from $U_{A}(1)$ anomaly in QCD. Namely the flavor singlet axial-vector current for light quarks has a nonzero divergence due to quantum anomaly effect. It is known that this effect is connected to the coupling of light quarks to a nontrivial topological configuration of gluon, i.e., instanton. The $U_{A}(1)$ anomaly term with spontaneous chiralsymmetry breaking, i.e., quark condensates, generates a diquark mass term, which behaves differently from the other mass terms. We discuss how we can identify and determine the parameters of such a coupling term of the diquark effective Lagrangian.

This paper is organized as follows. In Sec. II, we introduce diquarks and their local operator representation, and formulate chiral effective theory in the chiral-symmetry limit. In Sec. III, explicit chiral-symmetry breaking due to the quark masses is introduced and its consequences are discussed. In Sec. IV, a numerical estimate is given for the parameters of the effective theory. We use the diquark masses calculated in lattice QCD and also the experimental values of the singly heavy baryons. In Sec. V, a conclusion is given.

\section{DIQUARK EFFECTIVE THEORY}

\section{A. Diquark operators in flavor $S U(3)$ symmetry}

In order to study the transformation properties of the diquark systems, we consider properties of QCD composite operators made of two quark fields. We employ the flavor $S U$ (3) basis for the quark operators, $q_{\alpha, i}^{a}$, where $a$ is color, $\alpha$ is Dirac, $i$ is flavor index of the quark. Then local diquark operators are defined by

$$
\left(q_{i}^{T} \Gamma q_{j}\right)=\left(q_{\alpha, i}^{a}(\Gamma)_{\alpha \beta} q_{\beta, j}^{b}\right),
$$

where $T$ denotes the transpose for the Dirac index and $\Gamma$ is a relevant combination of the Dirac gamma matrices. Possible combinations satisfying the Lorentz covariance and the Pauli principle for quarks are given in Table I.

The first five operators belong to the total color $\overline{3}$ representation, i.e., color antisymmetric combinations, while the lower five are in the color symmetric 6
TABLE I. Local diquark operators. $C=i \gamma^{0} \gamma^{2}$ is the charge conjugation Dirac matrix. The rightmost column shows the relevant quantum states for two (nonrelativistic) quarks.

\begin{tabular}{llcccc}
\hline \hline & & $J^{\pi}$ & color & flavor & ${ }^{2 S+1} L_{J}$ \\
\hline 1 & $\left(q^{T} C q\right)_{A}^{\overline{3}}$ & $0^{-}$ & $\overline{3}$ & $\overline{3}$ & ${ }^{3} P_{0}$ \\
2 & $\left(q^{T} C \gamma^{5} q\right)_{A}^{\overline{3}}$ & $0^{+}$ & $\overline{3}$ & $\overline{3}$ & ${ }^{1} S_{0}$ \\
3 & $\left(q^{T} C \gamma^{\mu} \gamma^{5} q\right)_{A}^{\overline{3}}$ & $1^{-}$ & $\overline{3}$ & $\overline{3}$ & ${ }^{3} P_{1}$ \\
4 & $\left(q^{T} C \gamma^{\mu} q\right)_{S}^{\overline{3}}$ & $1^{+}$ & $\overline{3}$ & 6 & ${ }^{3} S_{1}$ \\
5 & $\left(q^{T} C \sigma^{\mu \nu} q\right)_{S}^{3}$ & $1^{+}, 1^{-}$ & $\overline{3}$ & 6 & ${ }^{3} D_{1},{ }^{1} P_{1}$ \\
6 & $\left(q^{T} C q\right)_{S}^{6}$ & $0^{-}$ & 6 & 6 & ${ }^{3} P_{0}$ \\
7 & $\left(q^{T} C \gamma^{5} q\right)_{S}^{6}$ & $0^{+}$ & 6 & 6 & ${ }^{1} S_{0}$ \\
8 & $\left(q^{T} C \gamma^{\mu} \gamma^{5} q\right)_{S}^{6}$ & $1^{-}$ & 6 & 6 & ${ }^{3} P_{1}$ \\
9 & $\left(q^{T} C \gamma^{\mu} q\right)_{A}^{6}$ & $1^{+}$ & 6 & $\overline{3}$ & ${ }^{3} S_{1}$ \\
10 & $\left(q^{T} C \sigma^{\mu \nu} q\right)_{A}^{6}$ & $1^{+}, 1^{-}$ & 6 & $\overline{3}$ & ${ }^{3} D_{1},{ }^{1} P_{1}$ \\
\hline \hline
\end{tabular}

representation. The indices $S$ (symmetric) and $A$ (antisymmetric) designate the flavor symmetry. In the last column, the corresponding nonrelativistic quark model assignments are given of the spin and orbital angular momentum.

\section{B. Scalar and pseudoscalar diquarks in chiral $S U(3)_{R} \times S U(3)_{L}$ symmetry}

In this paper, we concentrate on the scalar and pseudoscalar diquarks from the viewpoint of chiral symmetry. More specifically, we consider the first two states, Nos. 1 and 2 , from Table I, which have spin 0 , color $\overline{3}$ and flavor $\overline{3}$. We here see that these two diquarks are chiral partners to each other, i.e., they belong to the same chiral representation and therefore they would be degenerate if the chiral symmetry is not broken.

To see this, using the chiral projection operators, $P_{R, L} \equiv$ $\left(1 \pm \gamma_{5}\right) / 2$, we define the right quark, $q_{R, i}^{a}=P_{R} q_{i}^{a}$, as a $(3,1)$ representation of chiral $S U(3)_{R} \times S U(3)_{L}$ symmetry, and the left quark, $q_{L, i}^{a}=P_{L} q_{i}^{a}$, as a $(1,3)$ representation. Explicitly, they transform as

$$
\begin{array}{ll}
q_{R, i}^{a} \rightarrow\left(U_{R}\right)_{i j} q_{R, j}^{a}, & U_{R} \in S U(3)_{R} \\
q_{L, i}^{a} \rightarrow\left(U_{L}\right)_{i j} q_{L, j}^{a}, & U_{L} \in S U(3)_{L} .
\end{array}
$$

Then we construct "right" and "left" spin-0 diquark operators with color $\overline{3}$ as

$$
\begin{gathered}
d_{R, i}^{a} \equiv \epsilon^{a b c} \epsilon_{i j k}\left(q_{R, j}^{b T} C q_{R, k}^{c}\right), \\
d_{L, i}^{a} \equiv \epsilon^{a b c} \epsilon_{i j k}\left(q_{L, j}^{b T} C q_{L, k}^{c}\right) .
\end{gathered}
$$

It is straightforward to show that $d_{R}$ and $d_{L}$ belong to chiral $(\overline{3}, 1)$ and $(1, \overline{3})$ representation, respectively, and transform as 
TABLE II. Local diquark operators in the chiral basis.

\begin{tabular}{|c|c|c|c|}
\hline & spin & color & chiral \\
\hline$d_{R, i}^{a}=\epsilon_{a b c} \epsilon_{i j k}\left(q_{R, j}^{b T} C q_{R, k}^{c}\right)$ & 0 & $\overline{3}$ & $(\overline{3}, 1)$ \\
\hline$d_{L, i}^{a}=\epsilon_{a b c} \epsilon_{i j k}\left(q_{L, j}^{b T} C q_{L, k}^{c}\right)$ & 0 & $\overline{3}$ & $(1, \overline{3})$ \\
\hline$d_{(i, j)}^{a, \mu}=\epsilon_{a b c}\left(q_{L, i}^{b T} C \gamma^{\mu} q_{R, j}^{c}\right)$ & 1 & $\overline{3}$ & $(3,3)$ \\
\hline$d_{R\{i j\}}^{a, \mu \nu}=\epsilon_{a b c}\left(q_{R, i}^{b T} C \sigma^{\mu \nu} q_{R, j}^{c}\right)$ & 1 & $\overline{3}$ & $(6,1)$ \\
\hline$d_{L\{i j\}}^{a, \mu \nu}=\epsilon_{a b c}\left(q_{L, i}^{b T} C \sigma^{\mu \nu} q_{L, j}^{c}\right)$ & 1 & $\overline{3}$ & $(1,6)$ \\
\hline$\tilde{d}_{R\{i j\}}^{\{a b\}}=\left(q_{R, i}^{a T} C q_{R, j}^{b}+q_{R, j}^{a T} C q_{R, i}^{b}\right)$ & 0 & 6 & $(6,1)$ \\
\hline$\tilde{d}_{L\{i j\}}^{\{a b\}}=\left(q_{L, i}^{a T} C q_{L, j}^{b}+q_{L, j}^{a T} C q_{L, i}^{b}\right)$ & 0 & 6 & $(1,6)$ \\
\hline$\tilde{d}_{(i, j)}^{\{a b\}, \mu}=\left(q_{L, i}^{a T} C \gamma^{\mu} q_{R, j}^{b}+q_{L, i}^{b T} C \gamma^{\mu} q_{R, j}^{a}\right)$ & 1 & 6 & $(3,3)$ \\
\hline$\tilde{d}_{R, i}^{\{a b\}, \mu \nu}=\epsilon_{i j k}\left(q_{R, j}^{a T} C \sigma^{\mu \nu} q_{R, k}^{b}\right)$ & 1 & 6 & $(\overline{3}, 1)$ \\
\hline$\tilde{d}_{L, i}^{\{a b\}, \mu \nu}=\epsilon_{i j k}\left(q_{L, j}^{a T} C \sigma^{\mu \nu} q_{L, k}^{b}\right)$ & 1 & 6 & $(1, \overline{3})$ \\
\hline
\end{tabular}

The other diquark operators are also decomposed in the chiral basis similarly, as shown in Table II. First two of them are the diquarks that we concern in this paper.

The diquarks given above are not eigenstates of parity. Using the parity transform of the quark operators, $\mathcal{P}: q_{i R}^{a}(t, \boldsymbol{x}) \rightarrow \gamma^{0} q_{i L}^{a}(t,-\boldsymbol{x}), q_{i L}^{a}(t, \boldsymbol{x}) \rightarrow \gamma^{0} q_{i R}^{a}(t,-\boldsymbol{x})$, the spin-0 diquarks are found to transform as

$$
\mathcal{P}: d_{R, i}^{a} \rightarrow-d_{L, i}^{a}, \quad d_{L, i}^{a} \rightarrow-d_{R, i}^{a} .
$$

Thus the Lorentz scalar, $S\left(J^{\pi}=0^{+}\right)$, and pseudoscalar, $P$ $\left(0^{-}\right)$, operators can be identified by

$$
\begin{gathered}
S_{i}^{a}=\frac{1}{\sqrt{2}}\left(d_{R, i}^{a}-d_{L, i}^{a}\right)=\frac{1}{\sqrt{2}} \epsilon^{a b c} \epsilon_{i j k}\left(q_{j}^{b T} C \gamma_{5} q_{k}^{c}\right), \\
P_{i}^{a}=\frac{1}{\sqrt{2}}\left(d_{R, i}^{a}+d_{L, i}^{a}\right)=\frac{1}{\sqrt{2}} \epsilon^{a b c} \epsilon_{i j k}\left(q_{j}^{b T} C q_{k}^{c}\right) .
\end{gathered}
$$

These relations show that the scalar and pseudoscalar diquarks, given as Nos. 1 and 2 of Table I, belong to $(\overline{3}, 1)$ and $(1, \overline{3})$ representations of chiral symmetry. Thus, we conclude that the scalar and pseudoscalar diquarks are chiral partners.

\section{Chiral Lagrangian in the chiral limit}

We now introduce the chiral $(\overline{3}, 3)$ meson fields $\Sigma$, which contain nonet pseudoscalar and nonet scalar mesons. Their chiral transform is given by

$$
\Sigma_{i j} \equiv \sigma_{i j}+i \pi_{i j} \rightarrow U_{L, i k} \Sigma_{k m} U_{R, m j}^{\dagger}(\overline{3}, 3)
$$

where $\sigma$ represents a scalar nonet, and $\pi$ a pseudoscalar nonet. $\Sigma$ transforms under the spatial inversion as
$\mathcal{P}: \Sigma \rightarrow \Sigma^{\dagger}$. Chiral symmetry is spontaneously broken by the vacuum state, which is represented by the vacuum expectation value (VEV) of the scalar field $\sigma$ as

$$
\left\langle\Sigma_{i j}\right\rangle=\left\langle\sigma_{i j}\right\rangle=f \delta_{i j}, \quad\left\langle\pi_{i j}\right\rangle=0
$$

where $f$ is the pion decay constant. For this vacuum, $\pi_{i j}$ is the nonet of massless Nambu-Goldstone bosons. To read off the conventional meson contents from $\Sigma$, one uses the Gell-Mann matrices, $\lambda_{p}$, as

$$
\begin{gathered}
\Sigma_{i j} \equiv\left(\lambda_{p}\right)_{i j}\left(\sigma_{p}+i \pi_{p}\right) \\
\sigma_{p}=\frac{1}{4} \operatorname{Tr}\left[\lambda_{p}\left(\Sigma+\Sigma^{\dagger}\right)\right], \\
\pi_{p}=\frac{1}{4 i} \operatorname{Tr}\left[\lambda_{p}\left(\Sigma-\Sigma^{\dagger}\right)\right] .
\end{gathered}
$$

Note that $\sigma$ and $\pi$ contain the flavor singlet components, for which we use $\lambda_{0}=\sqrt{2 / 3} 1$ with the unit matrix 1 .

We are ready to present an effective Lagrangian in the chiral limit as

$$
\begin{aligned}
\mathcal{L}= & \mathcal{D}_{\mu} d_{R, i}\left(\mathcal{D}^{\mu} d_{R, i}\right)^{\dagger}+\mathcal{D}_{\mu} d_{L, i}\left(\mathcal{D}^{\mu} d_{L, i}\right)^{\dagger} \\
& -m_{0}^{2}\left(d_{R, i} d_{R, i}^{\dagger}+d_{L, i} d_{L, i}^{\dagger}\right) \\
& -\frac{m_{1}^{2}}{f}\left(d_{R, i} \Sigma_{i j}^{\dagger} d_{L, j}^{\dagger}+d_{L, i} \Sigma_{i j} d_{R, j}^{\dagger}\right) \\
& -\frac{m_{2}^{2}}{2 f^{2}} \epsilon_{i j k} \epsilon_{\ell m n}\left(d_{R, k} \Sigma_{\ell i} \Sigma_{m j} d_{L, n}^{\dagger}+d_{L, k} \Sigma_{\ell i}^{\dagger} \Sigma_{m j}^{\dagger} d_{R, n}^{\dagger}\right) \\
& +\frac{1}{4} \operatorname{Tr}\left[\partial^{\mu} \Sigma^{\dagger} \partial_{\mu} \Sigma\right]+V(\Sigma) .
\end{aligned}
$$

Here we truncate the interaction terms with more than two $\Sigma$ 's. We also omit $\Sigma \Sigma^{\dagger}$ - and $\Sigma^{\dagger} \Sigma$-terms, since they do not contribute to the mass difference between the $0^{+}$and $0^{-}$ states. $V(\Sigma)$, which is not shown explicitly, denotes the interaction potential terms for the meson fields that cause the spontaneous symmetry breaking, Eq. (12). Hereafter, we will omit the kinetic and potential parts of the mesons, because we consider only the mean fields of the mesons.

As the diquark is not a color-singlet state, we have introduced a color-gauge-covariant derivative in Eq. (16), $\mathcal{D}_{\mu}=\partial_{\mu}+i g T^{\alpha} G_{\mu}^{\alpha}$, with $G_{\mu}$ being the gluon field, and $T^{\alpha}$ the color $S U(3)$ generator for the $\overline{3}$ representation. All the color indices are contracted and not explicitly written. The kinetic energy term of the gluons fields is also omitted.

It is easy to check that the Lagrangian, Eq. (16), is chiral invariant and parity conserving. We may rewrite the Lagrangian in terms of the parity eigenstates, Eqs. (9) and (10), as 


$$
\begin{aligned}
\mathcal{L}= & \mathcal{D}_{\mu} S_{i}\left(\mathcal{D}^{\mu} S_{i}\right)^{\dagger}+\mathcal{D}_{\mu} P_{i}\left(\mathcal{D}^{\mu} P_{i}\right)^{\dagger} \\
& -m_{0}^{2}\left(S_{i} S_{i}^{\dagger}+P_{i} P_{i}^{\dagger}\right) \\
& -\frac{m_{1}^{2}}{f}\left(-S_{i} \sigma_{i j} S_{j}^{\dagger}+P_{i} \sigma_{i j} P_{j}^{\dagger}-i S_{i} \pi_{i j} P_{j}^{\dagger}+i P_{i} \pi_{i j} S_{j}^{\dagger}\right) \\
& -\frac{m_{2}^{2}}{2 f^{2}} \epsilon_{i j k} \epsilon_{\ell m n}\left[-S_{k}\left(\sigma_{\ell i} \sigma_{m j}-\pi_{\ell i} \pi_{m j}\right) S_{n}^{\dagger}\right. \\
& +P_{k}\left(\sigma_{\ell i} \sigma_{m j}-\pi_{\ell i} \pi_{m j}\right) P_{n}^{\dagger}+i S_{k}\left(\pi_{\ell i} \sigma_{m j}+\sigma_{\ell i} \pi_{m j}\right) P_{n}^{\dagger} \\
& \left.-i P_{k}\left(\pi_{\ell i} \sigma_{m j}+\sigma_{\ell i} \pi_{m j}\right) S_{n}^{\dagger}\right] .
\end{aligned}
$$

\section{Masses of the diquarks and generalized Goldberger-Treiman relation}

In the mean field approximation, keeping the $S U(3)$ symmetry, $\left\langle\Sigma_{i j}\right\rangle=f \delta_{i j}$, the masses of the diquarks are read from

$$
\begin{aligned}
\mathcal{L}_{\text {mass }}= & -m_{0}^{2}\left(d_{R, i} d_{R, i}^{\dagger}+d_{L, i} d_{L, i}^{\dagger}\right) \\
& -\left(m_{1}^{2}+m_{2}^{2}\right)\left(d_{R, i} d_{L, i}^{\dagger}+d_{L, i} d_{R, i}^{\dagger}\right),
\end{aligned}
$$

which leads to the mass matrix for $\left(d_{R, i}, d_{L, i}\right)$ as

$$
M^{2}=\left(\begin{array}{cc}
m_{0}^{2} & m_{1}^{2}+m_{2}^{2} \\
m_{1}^{2}+m_{2}^{2} & m_{0}^{2}
\end{array}\right)
$$

Diagonalizing the mass matrix, we obtain the mass eigenstates,

$$
\begin{aligned}
S_{i}^{a} & =\frac{1}{\sqrt{2}}\left(d_{R, i}^{a}-d_{L, i}^{a}\right) \\
& \longrightarrow M\left(0^{+}\right)=\sqrt{m_{0}^{2}-m_{1}^{2}-m_{2}^{2}}, \\
P_{i}^{a}= & \frac{1}{\sqrt{2}}\left(d_{R, i}^{a}+d_{L, i}^{a}\right) \\
& \longrightarrow M\left(0^{-}\right)=\sqrt{m_{0}^{2}+m_{1}^{2}+m_{2}^{2}},
\end{aligned}
$$

which are also the eigenstates of the parity.

Now it is clear how the diquark masses are generated from the spontaneous chiral-symmetry breaking (SCSB). In the regime of complete chiral restoration, the $0^{+}$and $0^{-}$ diquarks are degenerate with the mass $m_{0}$. This is the limit where all the hadrons are subjected to belong to a parity doublet. In the ordinary vacuum of QCD, the SCSB resolves their degeneracies. In the present case, the mass splitting is given by the $m_{1}^{2}$ - and $m_{2}^{2}$-terms.

It should be noted that the diquarks are bosons and their chiral behaviors are different from fermions. In the linear sigma model for baryons, if we assign the chirality of the baryon according to the chirality of quarks (naive choice), then the baryon mass should vanish in the chiral-symmetric limit. In this case, the baryon mass comes only from SCSB. It was, however, shown that the mirror assignment of chirality ( $L \leftrightarrow R$ reversed) of the baryon is possible, and then the chiral symmetric mass term is allowed. Realistic baryons may be a mixing of these two assignments [30-32].

In contrast, the chiral representation of the spin- 0 diquarks allows both the chiral symmetric and SCSB mass terms simultaneously. Namely, the $m_{0}^{2}$-term is independent from SCSB, while the $m_{1}^{2}$ - and $m_{2}^{2}$-terms contribute to the baryon masses only when the chiral symmetry is spontaneously broken.

As is shown in Eq. (17), the $m_{1}^{2}$ - and $m_{2}^{2}$-terms of the Lagrangian describe the meson-diquark interactions. The $\pi-S-P$ vertex terms are given by

$$
\begin{aligned}
\mathcal{L}_{\pi S P}= & \frac{i m_{1}^{2}}{f}\left(S_{i} \pi_{i j} P_{j}^{\dagger}-P_{i} \pi_{i j} S_{j}^{\dagger}\right) \\
& -\frac{i m_{2}^{2}}{f^{2}} \epsilon_{i j k} \epsilon_{\ell m n}\left(S_{k} \pi_{\ell i}\left\langle\sigma_{m j}\right\rangle P_{n}^{\dagger}-P_{k} \pi_{\ell i}\left\langle\sigma_{m j}\right\rangle S_{n}^{\dagger}\right) \\
= & \frac{i\left(m_{1}^{2}+m_{2}^{2}\right)}{f}\left(S_{i} \pi_{i j} P_{j}^{\dagger}-P_{i} \pi_{i j} S_{j}^{\dagger}\right) \\
& -\frac{i m_{2}^{2}}{f} \operatorname{Tr}[\pi]\left(S_{i} P_{i}^{\dagger}-P_{i} S_{i}^{\dagger}\right) \\
= & \frac{i\left(m_{1}^{2}+m_{2}^{2}\right)}{f} \pi_{p}\left(S \lambda_{p} P^{\dagger}-P \lambda_{p} S^{\dagger}\right) \\
& -\frac{3 i m_{2}^{2}}{f} \pi_{0}\left(S \lambda_{0} P^{\dagger}-P \lambda_{0} S^{\dagger}\right)
\end{aligned}
$$

where $\pi_{0}=\eta_{1}=\sqrt{6} \operatorname{Tr}[\pi]$ is the singlet pseudoscalar meson.

We then obtain the relation between the octet-mesondiquark couplings and the mass differences of diquarks, as a generalized Goldberger-Treiman (GT) relation,

$$
g_{\pi S P} \equiv \frac{m_{1}^{2}+m_{2}^{2}}{f}=\frac{M^{2}\left(0^{-}\right)-M^{2}\left(0^{+}\right)}{2 f} .
$$

This relation can also be derived from the conservation of the flavor-octet axial-vector currents and the existence of the massless Nambu-Goldstone bosons, $\pi_{p}(p=1, \ldots 8)$. Note that this coupling is a nonderivative, $S$-wave, coupling that describes the mesonic decay of the negative-parity excited heavy baryon into the positive-parity ground states.

On the other hand, the coupling constant of the singlet (eta) meson $\pi_{0}=\eta_{1}$ is given by

$$
g_{\pi_{0} S P}=\frac{m_{1}^{2}-2 m_{2}^{2}}{f} .
$$

This relation is not a GT relation and is not directly derived from the symmetry because the axial $U_{A}(1)$ is explicitly 

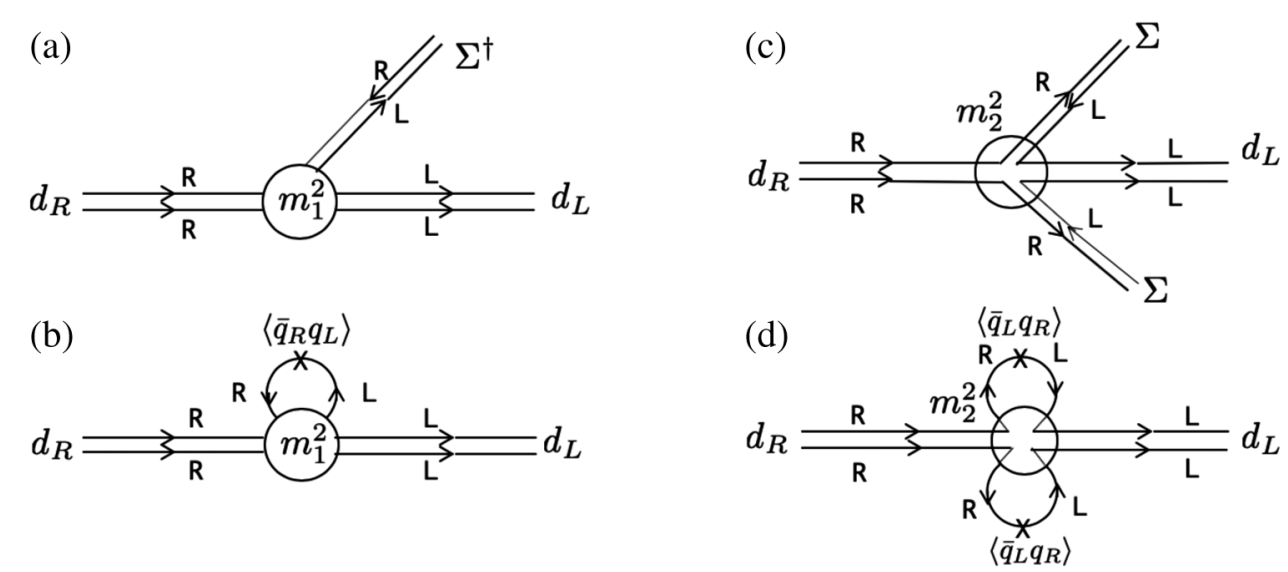

FIG. 1. Quark line representations of the diquark interaction terms. (a) $m_{1}^{2}$-term, (b) $m_{1}^{2}$-term with quark condensate, (c) $m_{2}^{2}$-term, and (d) $m_{2}^{2}$-term with quark condensate.

broken and the singlet $\eta_{1}$ is not a massless NambuGoldstone boson as is discussed in the next section.

\section{E. $U_{A}(1)$ anomaly}

So far, we have considered the chiral $S U(3)_{R} \times S U(3)_{L}$ symmetry. The given Lagrangian, Eq. (16), is invariant under the $S U(3)_{R} \times S U(3)_{L}$ transform. However, the QCD Lagrangian for massless quarks has another axial symmetry, $U_{A}(1)$ symmetry, which counts the difference of right and left quarks, regardless of flavor. In fact, this symmetry is not realized in the hadron spectrum due to anomaly. It is known that the instanton, a topologically nontrivial configuration of the gluon field in the Euclidean 4-dimensional space-time, plays a leading role in the $U_{A}(1)$ breaking. There the light quarks, $(u, d, s)$ couples to the instanton in an axial-symmetry breaking manner $[33,34]$.

In low-energy effective field theories, the $U_{A}(1)$ anomaly can be taken into account as an effective symmetry-breaking term. For instance, for the light meson sector, it is given as an extra term like $g_{D} \operatorname{det}\left(\Sigma+\Sigma^{\dagger}\right)$ (Kobayashi-Maskawa-'t Hooft term). ${ }^{1}$ It makes the flavor singlet $\eta_{1}\left(=\pi_{0}\right)$ massive, while the octet $\eta_{8}\left(=\pi_{8}\right)$ is massless in the chiral limit [35-37].

We similarly consider the $U_{A}(1)$ anomaly for the diquark effective theory. It happens that the $m_{1}^{2}$-term of Eq. (16),

$$
\mathcal{L}_{m 1}=-\frac{m_{1}^{2}}{f}\left(d_{R, i} \Sigma_{i j}^{\dagger} d_{L, j}^{\dagger}+d_{L, i} \Sigma_{i j} d_{R, i}^{\dagger}\right)
$$

breaks $U_{A}(1)$ symmetry [22,23]. It is easy to prove that each term contains three left and three right quarks with antisymmetric flavor indices. Thus the term is proportional to $\operatorname{det}_{i, j}\left(q_{R, j} q_{L, i}^{\dagger}+q_{L, j} q_{R, i}^{\dagger}\right)$. This is nothing but the determinant interaction, which is known to come from the instanton-light-quark couplings and breaks the $U_{A}(1)$

\footnotetext{
${ }^{1}$ We suppose that this term is included in the meson potential term in Eq. (16).
}

symmetry. In fact, by using the Fierz transformation, one can explicitly show

$$
\begin{aligned}
d_{R, i} \Sigma_{i j}^{\dagger} d_{L, j}^{\dagger} \propto & d_{R, i}^{b}\left(q_{R, i}^{a} \bar{q}_{L, j}^{a}\right) d_{L, j}^{b \dagger} \\
= & \epsilon_{b c d} \epsilon_{i p q}\left(q_{R, p}^{c T} C q_{R, q}^{d}\right) \\
& \times \epsilon_{b e f} \epsilon_{j r s}\left(q_{L, r}^{e T} C q_{L, s}^{f}\right)^{\dagger}\left(q_{R, i}^{a} \bar{q}_{L, j}^{a}\right) \\
= & 12 \operatorname{det}_{i j}\left(\bar{q}_{L, i}^{a} q_{R, j}^{a}\right) .
\end{aligned}
$$

It is clear that this term breaks $U_{A}(1)$ symmetry as the numbers of left and right quarks in each term are different. On the other hand, it keeps $S U(3)_{R} \times S U(3)_{L}$ invariant, because the flavor determinant is invariant under the $S U(3)$ transform.

Figure 1 shows the chiral properties of the vertices of the Lagrangian, Eq. (16). The $m_{1}^{2}$-term contains a six-quark vertex induced by the instanton, while the $m_{2}^{2}$-term does not break the $U_{A}(1)$ symmetry. The $U_{A}(1)$ anomaly effects will arise to the diquark mass and interaction only when the chiral symmetry is spontaneously broken.

\section{F. Masses of the singly heavy baryons}

As the diquarks are not directly observed, we may instead consider a singly heavy baryon, a bound state of a diquark with a heavy quark $Q$ (charm or bottom) [28,29]. Corresponding baryons are $\Lambda_{Q}$, and $\Xi_{Q}$ with spin-parity $1 / 2^{+}$and $1 / 2^{-}$. We write down the effective Lagrangian for the $Q q q$ baryons with the one-to-one correspondence to Eq. (16), as

$$
\begin{aligned}
\mathcal{L}_{Q q q \text { baryons }}= & \bar{S}_{R, i}(i v \cdot \partial) S_{R, i}+\bar{S}_{L, i}(i v \cdot \partial) S_{L, i} \\
& -M_{B 0}\left(\bar{S}_{R, i} S_{R, i}+\bar{S}_{L, i} S_{L, i}\right) \\
& -\frac{M_{B 1}}{f}\left(\bar{S}_{R, i} \Sigma_{i j}^{T} S_{L, j}+\bar{S}_{L, i} \Sigma_{i j}^{T \dagger} S_{R, j}\right) \\
& -\frac{M_{B 2}}{2 f^{2}} \epsilon_{i j k} \epsilon_{\ell m n}\left(\bar{S}_{L, k} \Sigma_{\ell i}^{T} \Sigma_{m j}^{T} S_{R, n}+\text { H.c. }\right),
\end{aligned}
$$


where $S_{L / R, i}$ denotes the effective field for the $Q q q$ baryon multiplets with the left/right $\overline{3}$ representation carrying the velocity $v^{\mu}$. We note that these $S_{L / R, i}$ fields are the heavy hadron effective fields for the fluctuation modes satisfying $v^{\mu} \gamma_{\mu} S_{L / R, i}=S_{L / R, i}{ }^{2}$. We may redefine the fields to eliminate the $M_{B 0}$-term as $S_{L / R, i} \rightarrow e^{-i M_{B 0} v^{\mu} x_{\mu}} S_{L / R, i}$, but we keep this term to see the explicit correspondence to the diquark Lagrangian in Eq. (16). The $M_{B 1}$-term is the one that breaks the $U_{A}(1)$ symmetry. This term generates anomalous mesonbaryon couplings, the $S$-wave $\Xi_{Q}\left(1 / 2^{-}\right) \Xi_{Q}\left(1 / 2^{+}\right) \pi$ and $\Xi_{Q}\left(1 / 2^{+}\right) \Lambda_{Q}\left(1 / 2^{-}\right) K$ couplings. The $M_{B 2^{-}}$-term is invariant under $U_{A}(1)$ symmetry transformation in addition to the chiral $S U(3)_{R} \times S U(3)_{L}$ symmetry transformation.

As in Eq. (12), the VEV of $\Sigma$ field causes the spontaneous chiral-symmetry breaking. Then, from the above Lagrangian, the masses of the baryons with positive and negative parities are given by

$$
\begin{aligned}
& M_{B+}=M_{Q}+M_{B 0}-M_{B 1}-M_{B 2}, \\
& M_{B-}=M_{Q}+M_{B 0}+M_{B 1}+M_{B 2},
\end{aligned}
$$

where $M_{Q}$ is the heavy-quark mass. As stated above, one can absorb $M_{B 0}$ into the redefinition of $M_{Q}$, which implies that it is impossible to distinguish $M_{B 0}$ and $M_{Q}$.

Now, let us assume that the binding energies between the heavy quark $Q$ and the diquark $q q$ are the same for the baryons with positive and negative parities. Then, the mass difference between two baryons is determined by the mass difference of the relevant diquarks. By comparing the formulas in Eq. (28) with the ones for diquark masses in Eqs. (20) and (21), the mass difference of chiral partners of the singly heavy baryons is related to the diquark mass parameter as

$$
\begin{aligned}
M_{B-}-M_{B+} & =M\left(0^{-}\right)-M\left(0^{+}\right) \\
& =\sqrt{m_{0}^{2}+m_{1}^{2}+m_{2}^{2}}-\sqrt{m_{0}^{2}-m_{1}^{2}-m_{2}^{2}} .
\end{aligned}
$$

\section{QUARK MASSES, $S U$ (3) BREAKING}

It is important to include the effects of the explicit breaking of chiral symmetry due to the quark masses, $\left(m_{u}, m_{d}, m_{s}\right)$, which are not zero nor equal. The mass hierarchies of the light mesons and baryons reflect the $S U$ (3) breaking due to the quark masses. In effective theories, this effect comes either in the choices of the

\footnotetext{
${ }^{2}$ The fields $S_{L / R, i}$ are related to the heavy baryon Dirac field operators $B_{L / R, i}$ as $B_{L / R, i}=\sum_{v^{\mu}} e^{-i M_{Q} v^{\mu} x_{\mu}} B_{L / R, i(v)}$ and $S_{L / R, i}=$ $P_{+} B_{L / R, i(v)}$, where $P_{+}$is the projection operator defined as $P_{+}=$ $\left(1+v^{\mu} \gamma_{\mu}\right) / 2$ and $M_{Q}$ is the heavy-quark mass.
}

parameters, or with extra terms with explicit breaking or both.

\section{A. Chiral Lagrangian with explicit symmetry breaking}

In the linear sigma model, we consider the effective quark mass generated by the current quark mass and the spontaneous chiral-symmetry breaking. Namely, with the condensation of $\sigma$, the quarks acquire an effective mass of $\sim 300-500 \mathrm{MeV}$.

$$
-m_{i} \bar{q}_{i} q_{i} \rightarrow-\left(m_{i}+g_{s}\left\langle\sigma_{i i}\right\rangle\right) \bar{q}_{i} q_{i}
$$

where $m_{i}$ is the current quark mass of the $i$ th flavor and $g_{s}(\sim 3)$ denotes the coupling of the scalar meson, $\sigma$, to the quark. We choose $\left\langle\sigma_{11}\right\rangle=\left\langle\sigma_{22}\right\rangle=f_{\pi} \sim 92 \mathrm{MeV}$ and $\left\langle\sigma_{33}\right\rangle=f_{s}=2 f_{K}-f_{\pi} \sim 128 \mathrm{MeV}$.

In a more general form, using the quark mass matrix, $\mathcal{M} \equiv \operatorname{diag}\left(m_{u}, m_{d}, m_{s}\right)$, and the VEV of $\Sigma$, we write the effective mass as

$$
\begin{gathered}
\mathcal{M}_{\mathrm{eff}}=\mathcal{M}+g_{s}\langle\Sigma\rangle \simeq\left(g_{s} f_{\pi}\right) \operatorname{diag}\{1,1, A\}, \\
A \equiv \frac{f_{s}}{f_{\pi}}\left(1+\frac{m_{s}}{g_{s} f_{s}}\right)>1 .
\end{gathered}
$$

Here we neglect $u$ and $d$ quark masses $\sim 2$ and $5 \mathrm{MeV}$, respectively, as they are much smaller than $g_{s} f_{\pi} \sim$ $300 \mathrm{MeV}$, while for $m_{s} \sim 100-200 \mathrm{MeV}, A \sim 5 / 3$ gives a significant correction.

Now we consider the symmetry breaking in the interaction terms of the Lagrangian, Eq. (16). The above consideration leads us to a prescription that the explicit symmetry breaking is introduced by the replacement,

$$
\Sigma \rightarrow \tilde{\Sigma} \equiv \Sigma+\mathcal{M} / g_{s} .
$$

This is justified because every mass insertion to a quark line in Feynman diagrams can have chiral-symmetry breaking $\langle\bar{q} q\rangle$ condensate in the same line.

Then this prescription gives the Lagrangian that includes explicit chiral-symmetry breaking as

$$
\begin{aligned}
\mathcal{L}_{\text {int }}= & -\frac{m_{1}^{2}}{f_{\pi}}\left(d_{R, i} \tilde{\Sigma}_{i j}^{\dagger} d_{L, j}^{\dagger}+d_{L, i} \tilde{\Sigma}_{i j} d_{R, j}^{\dagger}\right) \\
& -\frac{m_{2}^{2}}{2 f_{\pi}^{2}} \epsilon_{i j k} \epsilon_{\ell m n}\left(d_{R, k} \tilde{\Sigma}_{\ell i} \tilde{\Sigma}_{m j} d_{L, n}^{\dagger}+\text { H.c. }\right),
\end{aligned}
$$

with $\tilde{\Sigma}_{i j} \equiv \Sigma_{i j}+\frac{1}{g_{s}} \mathcal{M}_{i j}$.

\section{B. Diquark masses with $S U(3)$ breaking}

In the chiral-symmetry breaking vacuum, by replacing $\tilde{\Sigma}$ with its expectation value, $\langle\tilde{\Sigma}\rangle=\mathcal{M}_{\text {eff }} / g_{s}=$ $f_{\pi} \operatorname{diag}\{1,1, A\}$, in Eq. (34), we can read off the mass terms, as 


$$
\begin{aligned}
\mathcal{L}_{\text {mass }}= & -m_{0}^{2}\left(d_{R, i} d_{R, i}^{\dagger}+d_{L, i} d_{L, i}^{\dagger}\right) \\
& -\left(m_{1}^{2}+A m_{2}^{2}\right)\left(d_{R, 1} d_{L, 1}^{\dagger}+d_{L, 1} d_{R, 1}^{\dagger}\right. \\
& \left.+d_{R, 2} d_{L, 2}^{\dagger}+d_{L, 2} d_{R, 2}^{\dagger}\right) \\
& -\left(A m_{1}^{2}+m_{2}^{2}\right)\left(d_{R, 3} d_{L, 3}^{\dagger}+d_{L, 3} d_{R, 3}^{\dagger}\right) .
\end{aligned}
$$

The mass eigenstates are obtained by diagonalizing the mass matrix for each flavor,

$$
\begin{aligned}
\left(M^{2}\right)_{1,2} & =\left(\begin{array}{cc}
m_{0}^{2} & m_{1}^{2}+A m_{2}^{2} \\
m_{1}^{2}+A m_{2}^{2} & m_{0}^{2}
\end{array}\right), \\
\left(M^{2}\right)_{3} & =\left(\begin{array}{cc}
m_{0}^{2} & A m_{1}^{2}+m_{2}^{2} \\
A m_{1}^{2}+m_{2}^{2} & m_{0}^{2} .
\end{array}\right)
\end{aligned}
$$

The eigenstates coincide with the scalar $\left(S_{i}, 0^{+}\right)$and pseudoscalar $\left(P_{i}, 0^{-}\right)$diquarks again and their masses are given by

$$
\begin{gathered}
M_{1}\left(0^{+}\right)=M_{2}\left(0^{+}\right)=\sqrt{m_{0}^{2}-m_{1}^{2}-A m_{2}^{2}}, \\
M_{3}\left(0^{+}\right)=\sqrt{m_{0}^{2}-A m_{1}^{2}-m_{2}^{2}}, \\
M_{1}\left(0^{-}\right)=M_{2}\left(0^{-}\right)=\sqrt{m_{0}^{2}+m_{1}^{2}+A m_{2}^{2}}, \\
M_{3}\left(0^{-}\right)=\sqrt{m_{0}^{2}+A m_{1}^{2}+m_{2}^{2}} .
\end{gathered}
$$

Now one sees nontrivial hierarchy structures of the diquark masses. From Eqs. (38)-(41), one obtains

$$
\begin{aligned}
{\left[M_{1,2}\left(0^{+}\right)\right]^{2}-\left[M_{3}\left(0^{+}\right)\right]^{2} } & =\left[M_{3}\left(0^{-}\right)\right]^{2}-\left[M_{1,2}\left(0^{-}\right)\right]^{2} \\
& =(A-1)\left(m_{1}^{2}-m_{2}^{2}\right) .
\end{aligned}
$$

Note that the $i=1(d s)$ and $i=2(s u)$ diquarks are the ones with the strange quark, while the $i=3$ ( $u d$ ) diquark is nonstrange.

Suppose that the $(u d)$ scalar diquark is lighter than the $(d s)$ and $(s u)$ diquarks. This is a natural assumption which can be confirmed from the spectrum of the singly heavy baryons, $M\left(\Xi_{Q}=Q s u, Q d s\right)>M\left(\Lambda_{Q}=Q u d\right)$. As $M_{1}$ and $M_{2}$ correspond to the isodoublet diquarks, we need to have a mass hierarchy, $M_{1}\left(0^{+}\right)=M_{2}\left(0^{+}\right)>M_{3}\left(0^{+}\right)$. Now from Eq. (42) and $A>1, m_{1}^{2}>m_{2}^{2}$ is required. Then for the negative-parity diquarks, we will have $M_{1}\left(0^{-}\right)=$ $M_{2}\left(0^{-}\right)<M_{3}\left(0^{-}\right)$. This is an inverse hierarchy, because for the negative-parity diquarks, the strange ones $(i=1,2)$ are lighter than the nonstrange $(i=3)$ one.

\section{NUMERICAL ESTIMATES}

In order to determine the parameters of the effective Lagrangian, we need to have a few inputs. Ideally, the masses of the diquarks are useful. There are several attempts of computing the diquark masses and spectrum in lattice QCD [11-15]. As the diquark is not a color-singlet state, we need either fixing the gauge on the lattice and measure the diquark masses, or placing a heavy color source to compensate the color of the diquark and measure the mass (energy) differences of the different diquark states. Both the methods give qualitatively consistent results, in particular for the mass difference between the scalar diquark $\left(0^{+}\right)$and the axial-vector diquark $\left(1^{+}\right)$, which is about $150-200 \mathrm{MeV}$.

The other possible inputs are the masses of singly heavy baryons. The bound states of a spin- 0 diquark and a charm quark form charmed baryons, such as $\Lambda_{c}^{+}\left(c u d, 1 / 2^{ \pm}\right)$and $\Xi_{c}^{+, 0}$ (csu or $\left.c s d, 1 / 2^{ \pm}\right)$[38-44]. Assuming that the charm quark is a spectator, we can estimate the mass differences among the diquarks from those of the baryons.

Here we present two methods of determining the parameters, Method I (from lattice QCD) and II (from heavy baryon masses), in the following.

\section{A. Method I}

First, we take the diquark masses from a recent lattice QCD calculation for the Landau gauge in full QCD [15],

$$
\begin{gathered}
M_{3}\left(0^{+}\right)=725 \mathrm{MeV}, \\
M_{3}\left(0^{-}\right)=1265 \mathrm{MeV},
\end{gathered}
$$

$$
M_{1,2}\left(0^{+}\right)=906 \mathrm{MeV} .
$$

Then from Eq. (42), we obtain

$$
M_{1,2}\left(0^{-}\right)=1142 \mathrm{MeV} \text {. }
$$

By using the observed $\Lambda_{c}\left(1 / 2^{+}\right)$mass [given in Eq. (48)] as an input, we estimate the mass of $\Xi_{c}\left(1 / 2^{+}\right)$as

$$
\begin{aligned}
\left.M\left(\Xi_{c}, 1 / 2^{+}\right)\right|_{\text {theo }} & =M\left(\Lambda_{c}, 1 / 2^{+}\right)+\left(M_{1,2}\left(0^{+}\right)-M_{3}\left(0^{+}\right)\right) \\
& =2467 \mathrm{MeV}
\end{aligned}
$$

which beautifully agrees with the experimental value [given in Eq. (49)]. Similarly, the masses of $\Lambda_{c}\left(1 / 2^{-}\right)$and $\Xi_{c}\left(1 / 2^{-}\right)$are predicted as 


$$
\begin{aligned}
\left.M\left(\Lambda_{c}, 1 / 2^{-}\right)\right|_{\text {theo }} & =M\left(\Lambda_{c}, 1 / 2^{+}\right)+\left(M_{3}\left(0^{-}\right)-M_{3}\left(0^{+}\right)\right) \\
& =2826 \mathrm{MeV}, \\
\left.M\left(\Xi_{c}, 1 / 2^{-}\right)\right|_{\text {theo }} & =M\left(\Lambda_{c}, 1 / 2^{+}\right)+\left(M_{1,2}\left(0^{-}\right)-M_{3}\left(0^{+}\right)\right) \\
& =2704 \mathrm{MeV} .
\end{aligned}
$$

Note that the above results are independent from the choice of $A$. We, however, have to fix $A$ to determine the Lagrangian parameters $m_{1}^{2}$ and $m_{2}^{2}$. By assuming $A=5 / 3$,

$$
\begin{aligned}
& m_{0}^{2}=(1031 \mathrm{MeV})^{2}, \\
& m_{1}^{2}=(606.3 \mathrm{MeV})^{2}, \\
& m_{2}^{2}=-(274.4 \mathrm{MeV})^{2} .
\end{aligned}
$$

It should be noted here that the value of $m_{2}^{2}$ happens to be negative, but it is perfectly all right because this is a parameter of the Lagrangian whose sign is not constrained.

\section{B. Method II}

As an alternative, we may use the charmed baryon masses to determine the mass differences of the diquarks. In order to determine the diquark masses, we need the masses of $M\left(\Lambda_{c}, 1 / 2^{+}\right), M\left(\Xi_{c}, 1 / 2^{+}\right)$and $M\left(\Lambda_{c}, 1 / 2^{-}\right)$. The first two are experimentally given as [45]

$$
M\left(\Lambda_{c}, 1 / 2^{+}\right)=2286.46 \mathrm{MeV},
$$

$M\left(\Xi_{c}, 1 / 2^{+}\right)=\frac{1}{2}\left(M\left(\Xi_{c}^{+}\right)+M\left(\Xi_{c}^{0}\right)\right)=2469.42 \mathrm{MeV}$.

However, the masses of the negative-parity states have not been determined by experiment. $\Lambda_{c}\left(1 / 2^{-}\right)$observed at $2592 \mathrm{MeV}$ is not a $0^{-}$diquark bound state, but is rather a $P$-wave bound state of the $0^{+}$diquark (see discussions in Sec. IV C). Then the bound state of a $0^{-}$diquark and a charm quark is the second ( $\rho$-mode) $1 / 2^{-}$state and we do not have experimental data yet. Therefore we here use a quark model prediction of the second $\Lambda_{c}\left(1 / 2^{-}\right)$from Ref. [25],

$$
M\left(\Lambda_{c}, 1 / 2^{-}\right)=2890 \mathrm{MeV} .
$$

Using these data, we find

$$
\begin{aligned}
M_{1}\left(0^{+}\right)-M_{3}\left(0^{+}\right) & =M\left(\Xi_{c}, 1 / 2^{+}\right)-M\left(\Lambda_{c}, 1 / 2^{+}\right) \\
& =183 \mathrm{MeV} . \\
M_{3}\left(0^{-}\right)-M_{3}\left(0^{+}\right) & =M\left(\Lambda_{c}, 1 / 2^{-}\right)-M\left(\Lambda_{c}, 1 / 2^{+}\right) \\
& =604 \mathrm{MeV} .
\end{aligned}
$$

By using the lattice data for the lightest diquark mass as an input,

$$
M_{3}\left(0^{+}\right)=725 \mathrm{MeV},
$$

we obtain, from Eqs. (51), (52) and (42),

$$
\begin{gathered}
M_{1}\left(0^{+}\right)=906 \mathrm{MeV}, \\
M_{3}\left(0^{-}\right)=1329 \mathrm{MeV}, \\
M_{1}\left(0^{-}\right)=1212 \mathrm{MeV} .
\end{gathered}
$$

Then the masses of the $1 / 2^{-}$charmed baryons are predicted as

$$
\left.M\left(\Xi_{c}, 1 / 2^{-}\right)\right|_{\text {theo }}=2772 \mathrm{MeV} .
$$

Again, the above results are independent from the choice of $A$, while we can determine the parameters of the Lagrangian for the Method II, by setting $A=5 / 3$, as

$$
\begin{aligned}
& m_{0}^{2}=(1070 \mathrm{MeV})^{2}, \\
& m_{1}^{2}=(632 \mathrm{MeV})^{2}, \\
& m_{2}^{2}=-(213 \mathrm{MeV})^{2} .
\end{aligned}
$$

\section{Discussions}

The results obtained from the two methods are summarized in Table III. One immediately sees that the two methods give almost identical results. This simply indicates that our scheme works very well with the diquark masses given by the lattice QCD calculation.

A prominent feature of the mass spectrum is the inverse ordering of $\Lambda_{c}\left(1 / 2^{-}\right)$and $\Xi_{c}\left(1 / 2^{-}\right)$. This is anomalous from the quark model viewpoint because $\Xi_{c}=(\operatorname{csq})$ contains a strange quark and is expected to be heavier than $\Lambda_{c}(c q q)$ for the same quantum numbers. A naive estimate would conclude $M\left(\Xi_{c}\right) \sim M\left(\Lambda_{c}\right)+200(\mathrm{MeV})$,

TABLE III. Parameters of the chiral effective theory and the predicted diquark and baryon masses. The asterisk is for the input values. The experimental value of the $\Xi_{c}$ mass is the (charge) average of $\Xi_{c}^{0}$ and $\Xi_{c}^{+}$.

\begin{tabular}{lccc}
\hline \hline & Method I & Method II & Experiment \\
\hline$M_{3}\left(0^{+}\right)(\mathrm{MeV})$ & $725^{*}$ & $725^{*}$ & \\
$M_{1,2}\left(0^{+}\right)(\mathrm{MeV})$ & $906^{*}$ & 906 & \\
$M_{3}\left(0^{-}\right)(\mathrm{MeV})$ & $1265^{*}$ & 1329 & \\
$M_{1,2}\left(0^{-}\right)(\mathrm{MeV})$ & 1142 & 1212 & \\
$M\left(\Lambda_{c}, 1 / 2^{+}\right)(\mathrm{MeV})$ & $2286^{*}$ & $2286^{*}$ & 2286.46 \\
$M\left(\Xi_{c}, 1 / 2^{+}\right)(\mathrm{MeV})$ & 2467 & $2469^{*}$ & 2469.42 \\
$M\left(\Lambda_{c}, 1 / 2^{-}\right)(\mathrm{MeV})$ & 2826 & $2890^{*}$ & 2592 \\
$M\left(\Xi_{c}, 1 / 2^{-}\right)(\mathrm{MeV})$ & 2704 & 2772 & 2793 \\
$m_{0}^{2}\left(\mathrm{MeV}^{2}\right)$ & $(1031)^{2}$ & $(1070)^{2}$ & \\
$m_{1}^{2}\left(\mathrm{MeV}^{2}\right)$ & $(606)^{2}$ & $(631)^{2}$ & \\
$m_{2}^{2}\left(\mathrm{MeV}^{2}\right)$ & $-(274)^{2}$ & $-(210)^{2}$ & \\
\hline \hline
\end{tabular}


while the present chiral dynamics predicts $M\left(\Xi_{c}\right) \sim$ $M\left(\Lambda_{c}\right)-120(\mathrm{MeV})$ for the $1 / 2^{-}$states. The difference comes from the combination of the $U_{A}(1)$ anomaly term and the second-order chiral-symmetry breaking term as is seen in Eq. (42).

The PDG [45] reports a $\Lambda_{c}\left(1 / 2^{-}\right)$state at $2592 \mathrm{MeV}$ and a $\Xi_{c}\left(1 / 2^{-}\right)$state at $2793 \mathrm{MeV}$ (Table III). However, they may not directly be compared with our predictions. There are two competing structures for the negative parity $1 / 2^{-}$ baryon resonances, either a bound state of $0^{+}$diquark and a charm in $P$ wave $(L=1)\left(\lambda\right.$-mode), or a bound state of $0^{-}$ and a charm in $S$ wave ( $\rho$-mode). Our diquark picture assumes the $\rho$-mode excited states, where the diquark itself is excited. In the quark model analysis, the $\rho$-mode states are in general heavier than the $\lambda$-mode states [46]. In fact, $\Lambda_{c}(2592)$ fits to the $\lambda$-mode in the quark model very well [25].

On the other hand, because of the inverse ordering, our prediction of $\Xi_{c}\left(1 / 2^{-}\right)$comes as low as the observed state, $\Xi_{c}(2793)$, while, in the quark model, $\Xi_{c}(2793)$ would be assigned to the $\lambda$-mode excitation. It is interesting to see whether $\Xi_{c}(2793)$ is possible to be the $\rho$-mode excitation. If so, we expect to have two $\Xi_{c}\left(1 / 2^{-}\right)$states in the same energy region.

In the present numerical analysis, the parameter $A$ is fixed to $5 / 3 \sim 1.67$. This value comes from the conventional wisdom in the quark model that the ratio of the constituent quark masses of $s$ and $u / d$ is given by

$$
A=\frac{\mathcal{M}_{\mathrm{eff}}(s)}{\mathcal{M}_{\mathrm{eff}}(u / d)} \sim \frac{5}{3} .
$$

Let us estimate $A$ according to the definition, Eq. (32). First, $f_{s}=128 \mathrm{MeV}$, and $f_{\pi}=92 \mathrm{MeV}$ are determined from the weak decays of the pseudoscalar mesons. $g_{s}$ is the coupling constant of the pion to the $u, d$ quark. It can be related to the $\pi N N$ coupling constant, i.e., $g_{s}=$ $\frac{1}{3} g_{\pi N N} \sim 4.2$, Here we use $g_{\pi N N}=12.5$, which is determined from the Goldberger-Treiman relation. Finally $m_{s}$ is the current strange quark mass determined in the chiral perturbation theory, $m_{s} \sim 85-105 \mathrm{MeV}$ [45]. From these values, we obtain $A \sim 1.61-1.67$, which agrees with our choice.

While this estimate is plausible, we check how the results depend on the value of $A$. We recalculate the Lagrangian parameters for $A=1.5$ and 2 for the Method II. Noting that $m_{0}^{2}$ does not depend on $A$, we obtain

$$
\begin{aligned}
& m_{1}^{2}=(696 \mathrm{MeV})^{2}, \\
& m_{2}^{2}=-(328 \mathrm{MeV})^{2},
\end{aligned}
$$

for $A=1.5$ and
TABLE IV. $\quad A$ dependences of the parameters, $m_{1}^{2}$ and $m_{2}^{2}$ for the Method II.

\begin{tabular}{lcc}
\hline \hline$A$ & $m_{1}^{2}\left(\mathrm{MeV}^{2}\right)$ & $m_{2}^{2}\left(\mathrm{MeV}^{2}\right)$ \\
\hline 1.50 & $(696)^{2}$ & $-(328)^{2}$ \\
1.67 & $(631)^{2}$ & $-(210)^{2}$ \\
1.91 & $(569)^{2}$ & 0 \\
2.00 & $(552)^{2}$ & $(96)^{2}$ \\
\hline \hline & & \\
& & \\
& $m_{1}^{2}=(552 \mathrm{MeV})^{2}$, & \\
& $m_{2}^{2}=(96 \mathrm{MeV})^{2}$, &
\end{tabular}

for $A=2$.

We summarize the $A$ dependence in Table IV. It is found that the value of $m_{2}^{2}$ is sensitive to the choice of $A$. In fact, we can fit to the charmed baryon masses without $m_{2}^{2}$-term for $A=1.91$. On the other hand, the $U_{A}(1)$ anomaly term, $m_{1}^{2}$, is more stable and is consistently dominant.

\section{CONCLUSION}

In this paper, we have proposed a chiral effective theory of scalar and pseudoscalar diquarks. Based on the linear representations, we find that the color $\overline{3}$, flavor $\overline{3}$ and spinparity $0^{+}$diquark, $S$, and the $0^{-}$diquark, $P$, with the same color and flavor, form a chiral $(\overline{3}, 1)+(1, \overline{3})$ representation. Their mass difference comes from spontaneous chiral symmetry breaking (SCSB).

A linear-sigma-model Lagrangian is constructed with three mass parameters, $m_{0}^{2}, m_{1}^{2}$ and $m_{2}^{2}$. Among them, $m_{0}^{2}$ represents the chiral invariant mass. On the other hand, the $m_{1}^{2}$ - and $m_{2}^{2}$-terms yield masses under SCSB. Furthermore, the $m_{1}^{2}$-term breaks the axial $U_{A}(1)$ symmetry and thus represents the $U_{A}(1)$ anomaly. It is shown that the $m_{0}^{2}$ mass is diagonal in the chirality of the diquark, while the $m_{1}^{2}$ and $m_{2}^{2}$ masses are off-diagonal, connecting the left and right diquarks. The scalar and pseudoscalar diquarks are mass eigenstates and their mass difference is given by $m_{1}^{2}$ and $m_{2}^{2}$. We also find that the coupling of the pseudoscalar octet mesons to the diquarks satisfies the generalized Goldberger-Trieman relation.

By introducing the finite quark mass effects, we find that the contributions of the $U_{A}(1)$ anomaly mass $m_{1}^{2}$ depend on the quark mass in a reversed manner compared to the $m_{2}^{2}$ contributions. As a result, we find the inverse mass ordering of the negative-parity diquarks, $M\left(u s / d s, 0^{-}\right)<M\left(u d, 0^{-}\right)$.

In order to estimate the coupling constants in the effective Lagrangian, we take into account the results of lattice QCD calculations of diquark masses and also the masses of the bound states of a charm quark and a diquark, i.e., singly charmed baryons. We propose two methods of determining the parameters, which give similar results. The 
most prominent feature of the diquark picture of the charmed baryon is the reversed ordering of $\Lambda_{c}\left(1 / 2^{-}\right)$ and $\Xi_{c}\left(1 / 2^{-}\right)$. We predict a lower mass for $\Xi_{c}\left(1 / 2^{-}\right)$. This inversion is caused by the $U_{A}(1)$ anomaly term. A similar mass inversion was seen also in the scalar meson spectrum in a chiral effective theory approach [36].

Numerical values of the parameters in the effective Lagrangian show that the $U_{A}(1)$ anomaly term is dominant for the mass difference between the positive- and negativeparity diquarks.

So far, we have introduced only the scalar and pseudoscalar diquarks. It is interesting to extend this approach to vector and axial-vector diquarks. Considering finite temperature and baryon density is another direction to explore, as the diquark masses might change due to restoration of the chiral-symmetry breaking. These are subjects of future studies.

\section{ACKNOWLEDGMENTS}

We thank Dr. Daisuke Jido for useful discussions. This work was supported in part by JSPS KAKENHI Grants No. JP16K05345 (M. H.), No. JP17K14277 (K.S.), No. JP19H05159 (M. O.), and also by NNSFC (No. 11775132) (Y. R. L).
[1] H. X. Chen, W. Chen, X. Liu, and S. L. Zhu, The hiddencharm pentaquark and tetraquark states, Phys. Rep. 639, 1 (2016).

[2] A. Hosaka, T. Iijima, K. Miyabayashi, Y. Sakai, and S. Yasui, Exotic hadrons with heavy flavors: X, Y,Z, and related states, Prog. Theor. Exp. Phys. 2016, 062 C01 (2016).

[3] S. L. Olsen, T. Skwarnicki, and D. Zieminska, Nonstandard heavy mesons and baryons: Experimental evidence, Rev. Mod. Phys. 90, 015003 (2018).

[4] Y. R. Liu, H. X. Chen, W. Chen, X. Liu, and S. L. Zhu, Pentaquark and Tetraquark states, Prog. Part. Nucl. Phys. 107, 237 (2019).

[5] M. Ida and R. Kobayashi, Baryon resonances in a quark model, Prog. Theor. Phys. 36, 846 (1966).

[6] D. B. Lichtenberg and L. J. Tassie, Baryon mass splitting in a Boson-Fermion model, Phys. Rev. 155, 1601 (1967).

[7] M. Anselmino, E. Predazzi, S. Ekelin, S. Fredriksson, and D. B. Lichtenberg, Diquarks, Rev. Mod. Phys. 65, 1199 (1993).

[8] R. L. Jaffe and F. Wilczek, Diquarks and Exotic Spectroscopy, Phys. Rev. Lett. 91, 232003 (2003).

[9] R. L. Jaffe, Exotica, Phys. Rep. 409, 1 (2005).

[10] D. K. Hong, Y. J. Sohn, and I. Zahed, A diquark chiral effective theory and exotic baryons, Phys. Lett. B 596, 191 (2004).

[11] M. Hess, F. Karsch, E. Laermann, and I. Wetzorke, Diquark masses from lattice QCD, Phys. Rev. D 58, 111502 (1998).

[12] C. Alexandrou, P. de Forcrand, and B. Lucini, Evidence for Diquarks in Lattice QCD, Phys. Rev. Lett. 97, 222002 (2006).

[13] R. Babich, N. Garron, C. Hoelbling, J. Howard, L. Lellouch, and C. Rebbi, Diquark correlations in baryons on the lattice with overlap quarks, Phys. Rev. D 76, 074021 (2007).

[14] T. DeGrand, Z. Liu, and S. Schaefer, Diquark effects in light baryon correlators from lattice QCD, Phys. Rev. D 77, 034505 (2008).

[15] Y. Bi, H. Cai, Y. Chen, M. Gong, Z. Liu, H. X. Qiao, and Y. B. Yang, Diquark mass differences from unquenched lattice QCD, Chin. Phys. C 40, 073106 (2016).
[16] D. Ebert, T. Feldmann, C. Kettner, and H. Reinhardt, A diquark model for baryons containing one heavy quark, Z. Phys. C 71, 329 (1996).

[17] K. Nagata, A. Hosaka, and L. J. Abu-Raddad, The mass of the nucleon in a chiral quark-diquark model, Phys. Rev. C 72, 035208 (2005); Erratum, Phys. Rev. C 73, 049903 (2006).

[18] V. Dmitrašinović, K. Nagata, and A. Hosaka, Chiral properties of baryon interpolating fields, Mod. Phys. Lett. A 23, 2381 (2008).

[19] D. K. Hong and C. J. Song, Light scalar decay in diquark chiral effective theory, Int. J. Mod. Phys. A 27, 1250051 (2012).

[20] M. G. Alford, K. Rajagopal, and F. Wilczek, QCD at finite baryon density: Nucleon droplets and color superconductivity, Phys. Lett. B 422, 247 (1998).

[21] R. Rapp, T. Schäfer, E. V. Shuryak, and M. Velkovsky, Diquark Bose Condensates in High Density Matter and Instantons, Phys. Rev. Lett. 81, 53 (1998).

[22] T. Hatsuda, M. Tachibana, N. Yamamoto, and G. Baym, New Critical Point Induced by the Axial Anomaly in Dense QCD, Phys. Rev. Lett. 97, 122001 (2006).

[23] N. Yamamoto, M. Tachibana, T. Hatsuda, and G. Baym, Phase structure, collective modes, and the axial anomaly in dense QCD, Phys. Rev. D 76, 074001 (2007).

[24] K. Kim, D. Jido, and S. H. Lee, Diquarks: A QCD sum rule perspective, Phys. Rev. C 84, 025204 (2011).

[25] T. Yoshida, E. Hiyama, A. Hosaka, M. Oka, and K. Sadato, Spectrum of heavy baryons in the quark model, Phys. Rev. D 92, 114029 (2015).

[26] D. Jido and M. Sakashita, Quark confinement potential examined by excitation energy of the $\Lambda_{c}$ and $\Lambda_{b}$ baryons in a quark-diquark model, Prog. Theor. Exp. Phys. 2016, 083D02 (2016).

[27] K. Kumakawa and D. Jido, Excitation energy spectra of the $\Lambda_{c}$ and $\Lambda_{b}$ baryons in a finite-size diquark model, Prog. Theor. Exp. Phys. 2017, 123D01 (2017).

[28] Y. Kawakami and M. Harada, Analysis of $\Lambda_{c}(2595)$, $\Lambda_{c}(2625), \Lambda_{b}(5912), \Lambda_{b}(5920)$ based on a chiral partner structure, Phys. Rev. D 97, 114024 (2018). 
[29] Y. Kawakami and M. Harada, Singly heavy baryons with chiral partner structure in a three-flavor chiral model, Phys. Rev. D 99, 094016 (2019).

[30] C. E. Detar and T. Kunihiro, Linear sigma model with parity doubling, Phys. Rev. D 39, 2805 (1989).

[31] D. Jido, Y. Nemoto, M. Oka, and A. Hosaka, Chiral symmetry for positive and negative parity nucleons, Nucl. Phys. A671, 471 (2000).

[32] D. Jido, T. Hatsuda, and T. Kunihiro, Chiral Symmetry Realization for Even Parity and Odd Parity Baryon Resonances, Phys. Rev. Lett. 84, 3252 (2000).

[33] G. 't Hooft, Computation of the quantum effects due to a four-dimensional pseudoparticle, Phys. Rev. D 14, 3432 (1976); Erratum, Phys. Rev. D 18, 2199 (1978).

[34] G. 't Hooft, How instantons solve the U(1) problem, Phys. Rep. 142, 357 (1986).

[35] T. Hatsuda and T. Kunihiro, QCD phenomenology based on a chiral effective Lagrangian, Phys. Rep. 247, 221 (1994).

[36] Y. Kuroda, M. Harada, S. Matsuzaki, and D. Jido, Inverse mass hierarchy of light scalar mesons driven by anomalyinduced flavor breaking, arXiv:1910.09146.

[37] S. Kono, D. Jido, Y. Kuroda, and M. Harada, Role of $U_{A}(1)$ breaking term in dynamical chiral symmetry breaking of chiral effective theories, arXiv:1910.12982.

[38] L. Liu, H. W. Lin, K. Orginos, and A. Walker-Loud, Singly and doubly charmed $J=1 / 2$ baryon spectrum from lattice QCD, Phys. Rev. D 81, 094505 (2010).
[39] R. A. Briceno, H. W. Lin, and D. R. Bolton, Charmedbaryon spectroscopy from lattice QCD with $N_{f}=2+1+1$ flavors, Phys. Rev. D 86, 094504 (2012).

[40] Y. Namekawa et al. (PACS-CS Collaboration), Charmed baryons at the physical point in $2+1$ flavor lattice QCD, Phys. Rev. D 87, 094512 (2013).

[41] Z.S. Brown, W. Detmold, S. Meinel, and K. Orginos, Charmed bottom baryon spectroscopy from lattice QCD, Phys. Rev. D 90, 094507 (2014).

[42] P. Perez-Rubio, S. Collins, and G. S. Bali, Charmed baryon spectroscopy and light flavor symmetry from lattice QCD, Phys. Rev. D 92, 034504 (2015).

[43] C. Alexandrou and C. Kallidonis, Low-lying baryon masses using $N_{f}=2$ twisted mass clover-improved fermions directly at the physical pion mass, Phys. Rev. D 96, 034511 (2017).

[44] K. U. Can, H. Bahtiyar, G. Erkol, P. Gubler, M. Oka, and T. T. Takahashi, Spectrum of the Charmed Baryons in 2+1-flavor Lattice QCD, J. Phys. Soc. Jpn. Conf. Proc. 26, 022028 (2019).

[45] M. Tanabashi et al. (Particle Data Group), The review of particle physics, Phys. Rev. D 98, 030001 (2018), and 2019 update on-line http://pdg.lbl.gov.

[46] L. A. Copley, N. Isgur, and G. Karl, Charmed baryons in a quark model with hyperfine interactions, Phys. Rev. D 20, 768 (1979). 